\title{
Influence de la complémentation et de la prophylaxie sur la viabilité des ovins Foulbé au Nord-Cameroun
}

\author{
A. Njoya ${ }^{1}$ N.D. Awa ${ }^{1}$ D. Bouchel ${ }^{2}$
}

\section{Mots-clés}

O vin Foulbé - Brebis - Alimentation complémentaire - Contrôle de maladie Viabilité - Saison humide - Cameroun.

\begin{abstract}
Résumé
Q uatre lots de 60 agnelles âgées de 3 à 5 mois (lot CP : complémentation, vaccination contre la peste des petits ruminants, vermifugation et déparasitage externe ; lot $C$ : complémentation sans prophylaxie ni traitement; lot $P$ : prophylaxie et traitement sans complémentation ; lot $T$ : témoin) ont été constitués en avril 1993 en vue d'étudier l'effet de la complémentation alimentaire et des traitements sanitaires sur leur viabilité. La complémentation consistait en $200 \mathrm{~g}$ de tourteau de coton par tête et par jour et un complément minéral sous forme de pierre à lécher. Les traitements sanitaires consistaient en deux déparasitages internes en début et en fin des pluies et un déparasitage externe chaque fois que l'on notait la présence des tiques sur les agnelles. Les animaux ont fait l'objet d'un suivi clinique et de notation d'état corporel sur une année. Le jetage, les boiteries et les diarrhées ont représenté les principaux signes cliniques observés (respectivement 50,9, 15,2 et 13,0 p. 100). Les quotients annuels de mortalité ont été de $16,7,37,3,31,7$ et 52,5 p. 100 respectivement pour les lots $C P, C, P$ et $T$. La complémentation et la prophylaxie ont permis une réduction très sensible de la mortalité $(p<0,05)$. La mortalité a considérablement augmenté avec le retour des pluies. Les infestations par des parasites gastro-intestinaux et la sous-alimentation ont été les principaux facteurs favorisant la mortalité. Cette stratégie de complémentation protéique et de prophylaxie peut être encouragée pour réduire les fortes mortalités, principal facteur limitant la productivité des élevages ovins au Nord-Cameroun.
\end{abstract}

\section{INTRODUCTION}

L'élevage des petits ruminants est une activité génératrice d'importants revenus pour les paysans du Nord-Cameroun. Les ovins et les caprins constituent un capital rapidement mobilisable lorsqu'il faut faire face aux problèmes urgents de la famille tels que la santé ou la scolarité. Ils fournissent des protéines, des peaux et ont un grand rôle social et religieux. Malgré leur rôle capital dans l'économie des familles, très peu d'attention est accordée aux petits ruminants. Pendant la saison sèche, les troupeaux sont conduits aux pâturages par les enfants ou divaguent librement

1. Institut de recherches agricoles pour le développement, Station polyvalente de Garoua, BP 1073, Garoua, Cameroun

Tél. : (237) 272084 ; Fax (237) 271542

2. Laboratoire de recherches vétérinaires et zootechniques de Farcha, BP 433, N'Djamena, Tchad dans les villages. En saison des pluies, afin d'éviter les dégâts aux cultures, ils sont attachés au piquet ou en claustration dans une case ronde souvent insuffisamment aérée et nettoyée. En dehors de quelques résidus de récoltes, de déchets de cuisine et parfois de tourteau de coton, ils ne reçoivent généralement pas de complémentation alimentaire (1). A l'instar des bovins, aucun plan prophylactique n'est appliqué à ces espèces.

Schillhorn Van Veen et Ogunsusi (9) estiment qu'au Nord du Nigeria, les parasites internes et externes sont les principaux facteurs responsables des fortes mortalités des ovins. Les observations en milieu paysan au Nord-Cameroun (Martrenchar et coll., non publié) et au Tchad (4) incriminent plutôt les pneumopathies comme cause majeure de mortalité. Des taux de mortalité de près de 50 p. 100 en élevages ovins ont été enregistrés au NordCameroun malgré une complémentation à base de tourteau de coton (1). 
L'étude du contrôle de la mortalité des ovins s'est souvent limitée soit à des essais de prophylaxie sanitaire, soit à de complémentations alimentaires. Ce type d'approche a montré ses limites $(1,2)$. On ne saurait d'ailleurs incriminer un facteur spécifique. En effet, une combinaison de plusieurs facteurs peut être responsable de la morbidité et de la mortalité des ovins. De fait, la synergie de deux ou plusieurs facteurs (génétique, statut nutritionnel et physiologique, facteurs climatiques et environnementaux) peut entraîner l'apparition et le développement de pathologies parfois fatales $(3,4)$. L'objectif de cette étude était d'évaluer les relations observées entre des indicateurs nutritionnels, l'état sanitaire et les paramètres climatiques d'une part et la mortalité d'autre part, ainsi que l'influence d'une complémentation et d'un programme de prophylaxie sur la viabilité des agnelles.

\section{- MATERIEL ET METHODES}

\section{Site de l'étude}

L'étude a été menée à l'Institut de recherche agricole pour le développement (IRAD), station polyvalente de Garoua, située à la latitude E de 9,3 $\mathrm{N}$ au Nord-Cameroun, d'avril 1993 à avril 1994. La pluviométrie annuelle avoisine $990 \mathrm{~mm}$ avec près de 50 p. $100 \mathrm{de}$ pluies enregistrées entre juillet et août. La pluviométrie a été normale pendant la période d'étude ( $946 \mathrm{~mm} / \mathrm{an})$. L'année est divisée en trois saisons : une saison sèche chaude de février à mai, une saison des pluies de juin à septembre et une saison sèche froide d'octobre à janvier. Le pâturage est naturel avec des graminées pérennes dominantes comme Andropogon gayanus, Hyparrhenia rufa, Cynodon dactylon, Spermacoa stachydea, Jardinia congoensis et Heteropogon contortus. Des arbres et arbustes contribuent aussi à la biomasse consommable par les ovins, particulièrement pendant la saison sèche. Les plus communs sont Daniellia oliveri, Afzelia africana, Prosopis africana, Ficus spp., Grewia spp., Stereospermum kunthianum, Crossopterix febrifuga et Vitex simplex (Njoya et Awa, non publié).

\section{Animaux}

En janvier et février 1993, 350 agnelles de race locale Foulbé, âgées de 3 à 4 mois ont été achetées juste après le sevrage. Pendant la période de quarantaine, elles ont été identifiées avec des boucles auriculaires (Allflex ${ }^{\mathrm{ND}}$ ), vermifugées avec du Valbazen ${ }^{N D}$ (fenbendazole) et un déparasitage externe avec l'Ectopor ${ }^{\mathrm{ND}}$ (diazinon). A l'issue d'une triple pesée, 240 agnelles ont été retenues en avril 1993, sur la base du poids, de l'âge et du phénotype ovin Foulbé. Elles ont été réparties en 60 blocs de quatre agnelles par stratification sur la base de leur poids et de leur note d'état corporel (NEC). Les agnelles de chaque bloc ont été ensuite affectées de façon aléatoire dans quatre lots, de manière à avoir des lots homogènes de 60 animaux chacun.

La NEC était déterminée par palpation des régions dorsale, lombaire et autour de la queue, et par appréciation de l'aspect général de l'animal sur une grille allant de 1 à 4 , avec des écarts de 0,25.

\section{Traitements expérimentaux}

$\mathrm{Au}$ premier lot $(\mathrm{CP})$, un complément alimentaire était distribué et des traitements prophylactiques et curatifs administrés. La complémentation consistait en $200 \mathrm{~g}$ de tourteau de coton (40,8 p. 100 de matières azotées totales/matière brute) par agnelle et par jour, distribuée en groupe, et en une complémentation minérale sous forme de pierre à lécher $(55$ p. 100 de poudre d'os, 40 p. 100 de sel, 4 p. 100 de complément minéral et vitaminé et 1 p. 100 de ciment) à disposition en permanence dans le parc de nuit. La prophylaxie consistait en deux déparasitages internes contre les helminthoses gastro-intestinales en début et en fin des pluies (respectivement mai et octobre ) et une vaccination contre la peste des petits ruminants (PPR) effectuée en fin de saison de pluies (octobre) à l'aide du Bovipestovax ${ }^{\mathrm{ND}}$ (souche dirigée contre la peste bovine produite par le Laboratoire national vétérinaire de Boklé, Garoua). Un déparasitage externe était effectué chaque fois que l'on notait la présence de tiques sur les agnelles. Le second lot (C) avait la complémentation seule, le troisième lot $(\mathrm{P})$ n'était pas complémenté, mais recevait les traitements prophylactiques et antiparasitaires externes. Le dernier lot (T) servait de témoin.

Les soins légers étaient administrés aux agnelles des lots $\mathrm{CP}$ et $\mathrm{P}$ lorsqu'elles étaient malades.

\section{Conduite des troupeaux}

Les quatre lots étaient élevés séparément, gardés chacun par un berger ; cependant une rotation des lots dans les quatre bergeries et dans le gardiennage était effectuée tous les mois afin d'annuler les effets « berger et bergerie » dans l'analyse statistique des données. Les bergeries étaient simples, faites de poteaux supportant une toiture en tôles à deux pentes. Une natte en paille tissée ou secco montant à une hauteur de $0,90 \mathrm{~m} \mathrm{du}$ sol, disposée autour de la bergerie, protégeait les animaux contre le vent. Le sol était en terre battue afin de favoriser l'infiltration des urines et le nettoyage des fèces. La pâture sur savane naturelle durait $7 \mathrm{~h}$ par jour, entrecoupée par deux abreuvements à 12 et $16 \mathrm{~h}$.

\section{Collecte des données}

Le poids et la NEC des agnelles étaient collectés mensuellement sur tous les animaux. Les principaux signes cliniques observés lors des visites quotidiennes étaient enregistrés. Les mortalités et leurs causes possibles étaient notées. Les données climatiques provenaient de la station de météorologie de l'IRAD, à 1 km de distance.

\section{Prélèvements sanguins}

Du sang était prélevé à la veine jugulaire mensuellement durant les 12 mois d'observation sur les mêmes 20 agnelles par lot (tirées aléatoirement) dans deux tubes. Les prélèvements de sang dans les tubes au fluorure de sodium servaient à déterminer la teneur en glucose (indicateur des carences énergétiques). Les tubes avec l'EDTA servaient à déterminer l'urée (indicateur des carences azotées), l'hématocrite et l'hémoglobine (indicateurs de l'anémie). Suite aux mortalités intervenues dans les différents lots, 1180 échantillons de sang ont été collectés pendant l'étude.

Les analyses de glucose, d'urée et d'hémoglobine étaient effectuées par spectrophotométrie sur analyseur automatique Coulter ${ }^{\mathrm{ND}}$ à l'aide de kits (BioMérieux $\left.{ }^{\mathrm{ND}}\right)$. Le taux d'hématocrite était déterminé en utilisant une centrifugeuse à hématocrite.

\section{Analyse statistique des données}

La corrélation de Pearson a été utilisée pour évaluer les relations entre l'occurrence des principaux signes cliniques, les paramètres climatiques et la mortalité. La régression linéaire multiple (méthode pas à pas) a été effectuée pour évaluer l'importance des différentes variables dans le modèle expliquant la mortalité :

$$
\mathrm{M}=\alpha \mathrm{H}+\beta \mathrm{P}+\gamma \mathrm{T}+\lambda \mathrm{D}+\delta \mathrm{J}+\chi \mathrm{D}+\mu
$$


où $\mathrm{M}$ est le nombre de cas de mortalité, $\mathrm{H}$ est l'humidité relative en pourcentage, $\mathrm{P}$ est la pluviométrie en $\mathrm{mm}$, T est la température moyenne en ${ }^{\circ} \mathrm{C}$, D est la morbidité globale, J est le nombre de cas de jetage, et $\mathrm{D}$ est le nombre de cas de diarrhée. Les données ont été analysées par la procédure « Reg » du logiciel statistique SAS (8). Les quotients annuels de mortalité, correspondant au nombre de cas de mortalité au cours de l'année par rapport à l'effectif initial du lot, ont été comparés par le test de Chi-2.

\section{RESU LTATS}

\section{Statut sanitaire des animaux des lots expérimentaux}

Au cours des 12 mois d'étude, 401 cas cliniques ont été enregistrés (tableau I). La morbidité globale était plus faible dans le lot $\mathrm{P}$ (62 cas seulement sur un total de 401 cas) que dans les trois autres lots. Le lot $\mathrm{C}$ avait le plus grand nombre de cas de morbidité (32 p. 100 de cas). Jetage, boiterie, lésions cutanées associées au prurit et diarrhée ont constitué les principaux symptômes cliniques observés et ont représenté respectivement 50,9, 15,2, 14,0 et 13,0 p. 100 des cas de morbidité. La comparaison de la fréquence jetage et de diarrhées n'a pas révélé de différence significative entre lots (tableau I).

De nombreux cas de boiterie ont été enregistrés dans les lots $C$ et $\mathrm{T}$ (65,6 p. 100 de cas), surtout en saison pluvieuse.

Une épidémie de gale a été observée dans le lot C (73 p. 100 du nombre total de cas) (tableau I). Au cours du mois de janvier, 29 cas ont été enregistrés.

Le quotient annuel de mortalité était de 16,7, 37,3, 31,7 et 52,5 p. 100 respectivement dans les lots CP, C, P et T. Il était statistiquement beaucoup plus élevé $(\mathrm{p}<0,05)$ dans le lot $\mathrm{T}$ que dans les trois lots expérimentaux (tableau I). Le minimum de cas de mortalité a été enregistré dans le lot CP (10 cas), alors que le maximum l'a été dans le lot $\mathrm{T}$ (31 cas).

\section{Influence des facteurs climatiques sur la morbidité et la mortalité des agnelles}

Sur l'ensemble des agnelles des quatre lots, une analyse des fréquences mensuelles a fait ressortir deux pics d'apparition de jetage : un premier pic culminant en saison sèche froide (janvier) et un second en saison des pluies (juillet). La prévalence du jetage a été plus marquée de décembre à mars (saison sèche), avec 145 sur les 204 cas (71 p. 100) enregistrés au cours de l'étude (tableau II).

Deux périodes où la fréquence des diarrhées a été plus importante ont été identifiées en saison des pluies (juillet-août) avec 44 p. 100 des cas enregistrés, et en saison sèche chaude (mars-mai) caractérisée par les premières pluies, avec 29 p. 100 de cas (tableau II).

Près de 63 p. 100 des mortalités ont eu lieu pendant la saison des pluies et le début de la saison sèche, de juin à octobre (tableau II).

On a noté une corrélation significative $(r=-0,62 ; \mathrm{p}<0,05)$ entre la fréquence d'apparition de jetage et le taux d'humidité relative, et la température $(\mathrm{r}=-0,50 ; \mathrm{p}<0,05)$ (tableau III).

Une corrélation positive significative a également été observée entre la fréquence des mortalités et l'humidité relative $(\mathrm{r}=0,67$; $\mathrm{p}=0,02)$ ainsi que la pluviométrie $(\mathrm{r}=0,67 ; \mathrm{p}=0,02)$. Une corrélation négative a été notée entre la fréquence des mortalités et la température $(\mathrm{r}=-0,63 ; \mathrm{p}=0,03)$ (tableau III). La régression par la procédure pas à pas des facteurs associés à la mortalité a montré que, dans 70 p. 100 de cas, le nombre de cas de mortalité (M) augmentait avec l'augmentation du taux d'humidité et de cas de morbidité, suivant la relation :

$$
\begin{gathered}
\mathrm{M}=0,114 * \text { Humidité }+0,085 * \text { Morbidités }-3,16 \\
\left(\mathrm{r}^{2}=0,70 ; \mathrm{p}=0,02\right)
\end{gathered}
$$

\section{Relation entre la note d'état corporel et la mortalité des agnelles}

La NEC moyenne des agnelles des quatre lots était de 2,80 au début et 2,92 à la fin de l'essai. Les 82 agnelles mortes au cours de l'étude ont connu une chute de leur NEC, soit une note moyenne de 2,26 en moyenne avant leur mort (tableau IV). La NEC précédant la mort par rapport à leur état initial était statistiquement plus faible $(\mathrm{p}<0,05)$ pour le lot $\mathrm{P}(2,24)$ et le lot $\mathrm{T}(2,06)$, ces deux lots ne recevant pas de complémentation alimentaire, contrairement aux lots CP $(2,55)$ et $\mathrm{C}(2,40)$.

Les agnelles survivantes du lot T ont connu une chute de leur NEC au cours de l'étude ( $\mathrm{p}<0,05)$, passant de 2,82 à 2,30 contrairement aux agnelles des trois autres lots qui ont maintenu ou amélioré leur NEC (tableau IV).

L'évolution pondérale des agnelles (tableau IV) a été influencée significativement par la complémentation ( $p<0,0001)$. En avril 1994, les agnelles complémentées avaient un poids moyen supérieur de 35 p. 100 à celui des animaux ne recevant pas de complémentation. Les traitements prophylactiques n'ont pas affecté l'évolution pondérale au cours de l'étude. La corrélation entre le poids et la NEC en fin d'essai n'était pas significative $(\mathrm{r}=0,46$; $\mathrm{p}>0,1)$.

\section{Relation entre certains paramètres métaboliques et la mortalité des agnelles}

Pendant toute l'étude, la complémentation a été associée à une augmentation significative de la teneur plasmatique en urée $(\mathrm{p}<0,0001)$ et en glucose $(\mathrm{p}<0,01)$. Ces teneurs (tableau V) étaient plus élevées en saison des pluies qu'au cours des autres saisons. La teneur en hémoglobine a faiblement varié en fonction des traitements. Cependant, le taux d'hématocrite du lot $\mathrm{T}$ était faible en comparaison des lots CP, P et C. Au cours de l'étude, des corrélations positives ont été observées entre les teneurs en urée et en glucose $(\mathrm{r}=0,38 ; \mathrm{p}<0,01)$, et entre l'hématocite et l'hémoglobine $(r=0,74 ; p<0,001)$.

En s'appuyant sur les valeurs enregistrées lors du prélèvement de sang précédant la mort, les teneurs en urée plasmatique n'étaient pas statistiquement différentes $(\mathrm{p}>0,05)$ entre les agnelles des quatre lots mortes par rapport aux valeurs observées chez les agnelles vivantes (tableau V). Les valeurs extrêmes étaient de 0,10 et $0,29 \mathrm{~g} / \mathrm{l}$ respectivement chez les agnelles mortes des lots $\mathrm{P}$ et $\mathrm{C}$, et de 0,25 et $0,32 \mathrm{~g} / \mathrm{l}$ chez les agnelles vivantes des lots $\mathrm{T}$ et $\mathrm{C}$.

La teneur en glucose plasmatique était sensiblement la même dans les quatre lots d'agnelles vivantes. Chez les agnelles des lots $\mathrm{P}$ et $\mathrm{T}$ peu avant leur mort, elle était respectivement de 0,47 et $0,45 \mathrm{~g} / \mathrm{l}$, contre $0,60 \mathrm{~g} / \mathrm{l}$ pour les agnelles vivantes dans ces lots.

Les taux d'hémoglobine et d'hématocrite enregistrés chez les agnelles peu avant leur mort étaient significativement plus faibles $(\mathrm{p}<0,05)$ par rapport aux valeurs observées chez les agnelles vivantes dans les quatre lots (tableau V). Globalement le taux d'hémoglobine était inférieur à 7 p. 100 peu avant leur mort, contre une valeur de plus de 8,6 p. 100 chez les agnelles vivantes des quatre lots. Celui d'hématocrite était inférieur à 16 p. 100 peu avant leur mort, contre une valeur de plus de 26 p. 100 chez les agnelles vivantes des quatre lots. 
Feed supplementation and prophylactic treatment of sheep in Cameroon

\section{Tableau I}

Repartition des signes cliniques et des mortalités observés selon les lots

\begin{tabular}{|c|c|c|c|c|c|c|}
\hline Lot & $\mathrm{CP}^{1}$ & $C^{2}$ & $\mathbf{P}^{3}$ & $\mathbf{T}^{4}$ & Total & $\%$ \\
\hline Signes cliniques & 111 & 129 & 62 & 99 & 401 & \\
\hline Jetage & 71 & 51 & 34 & 48 & 204 & 50,87 \\
\hline Diarrhées & 10 & 15 & 12 & 15 & 52 & 12,97 \\
\hline Boiteries & 11 & 16 & 10 & 24 & 61 & 15,21 \\
\hline Lésions cutanées et prurit & 10 & 41 & 1 & 4 & 56 & 13,97 \\
\hline Ecthyma contagieux & 9 & 6 & 5 & 8 & 28 & 6,98 \\
\hline Effectif initial des agnelles & 60 & 60 & 60 & 60 & 240 & \\
\hline Nombre de mortalités & 10 & 22 & 19 & 31 & & \\
\hline Quotient annuel de mortalité, \% & $16,7^{a}$ & $37,3^{b}$ & $31,7^{c}$ & $52,5^{d}$ & & \\
\hline
\end{tabular}

${ }^{1}$ Complémentation alimentaire et traitements prophylactiques

${ }^{2}$ Complémentation alimentaire sans traitements prophylactiques

${ }^{3}$ Traitements prophylactiques uniquement

${ }^{4}$ Témoin

${ }^{\text {abcd } L e s ~ m o y e n n e s ~ s u r ~ l a ~ m e ̂ m e ~ l i g n e ~ s u i v i e s ~ d e ~ l e t t r e s ~ d i f f e ́ r e n t e s ~ s o n t ~ s i g n i f i c a t i v e m e n t ~ d i f f e ́ r e n t e s ~ a u ~ s e u i l ~ d e ~} 5 \%$

Tableau II

Paramètres climatiques et fréquence des pathologies majeures au cours d'une année

$\begin{array}{lccccccc}\text { Mois } & \begin{array}{c}\text { Température } \\ \text { moyenne, }{ }^{\circ} \mathrm{C}\end{array} & \begin{array}{c}\text { Humidité } \\ \text { relative, } \%\end{array} & \begin{array}{c}\text { Pluviométrie } \\ \mathrm{mm}\end{array} & \begin{array}{c}\text { Effectif des } \\ \text { agnelles à } \\ \text { la fin du mois }\end{array} & \begin{array}{c}\mathrm{Nb} \text {. de cas } \\ \text { de jetage }\end{array} & \begin{array}{c}\mathrm{Nb} \text {. de cas } \\ \text { de diarrhées }\end{array} & \begin{array}{c}\mathrm{Nb} \text {. de cas } \\ \text { de mortalité }\end{array}\end{array}$

\begin{tabular}{|c|c|c|c|c|c|c|c|}
\hline Avril 93 & 32,4 & 47 & 92 & 236 & 5 & 5 & 4 \\
\hline Mai 93 & 29,3 & 80 & 139 & 233 & 11 & 3 & 3 \\
\hline Juin 93 & 27,5 & 87 & 157 & 226 & 1 & 1 & 7 \\
\hline Juillet 93 & 26,1 & 93 & 218 & 215 & 24 & 15 & 11 \\
\hline Août 93 & 25,8 & 96 & 293 & 203 & 5 & 8 & 12 \\
\hline Septembre 93 & 25,8 & 96 & 205 & 193 & 7 & 1 & 10 \\
\hline Octobre 93 & 27,2 & 88 & 118 & 181 & 6 & 3 & 12 \\
\hline Novembre 93 & 28,7 & 60 & 0 & 176 & 11 & 4 & 5 \\
\hline Décembre 93 & 27,0 & 35 & 0 & 173 & 21 & 1 & 3 \\
\hline Janvier 94 & 25,1 & 24 & 0 & 164 & 64 & 2 & 9 \\
\hline Février 94 & 28,8 & 15 & 0 & 163 & 23 & 2 & 1 \\
\hline Mars 94 & 32,1 & 31 & 0 & 158 & 26 & 7 & 5 \\
\hline
\end{tabular}

\section{Tableau III}

Correlation de Pearson entre les variables climatiques et l'occurence des pathologies majeures

\begin{tabular}{lccccrr} 
& Température & Humidité & Pluviométrie & Jetage & Diarrhées & Mortalité \\
\hline Température & 1,00 & $-0,387$ & $-0,397$ & $-0,248$ & 0,017 & $-0,631$ \\
Humidité & & 1,00 & 0,885 & $-0,617$ & 0,287 & 0,673 \\
Pluviométrie & & & 1,00 & $-0,503$ & 0,413 & 0,665 \\
Jetage & & & 1,00 & 0,027 & $-0,029$ \\
Diarrhées & & & & 1,00 & 0,380 \\
Mortalité & & & & & & 1,00
\end{tabular}


Tableau IV

Comparaisons de la note d'état corporel (NEC) des agnelles au cours de l'essai et peu avant la mort

\begin{tabular}{|c|c|c|c|c|c|c|c|c|c|}
\hline \multirow[b]{2}{*}{ Lot } & \multicolumn{3}{|c|}{ NEC initiale (avril 1993) } & \multicolumn{3}{|c|}{ NEC finale (avril 1994) } & \multicolumn{3}{|c|}{ NEC peu avant la mort } \\
\hline & $\begin{array}{c}\text { Nb. } \\
\text { d'agnelles }\end{array}$ & Moy. & E.T. ${ }^{1}$ & $\begin{array}{c}\mathrm{Nb} \text {. } \\
\text { d'agnelles }\end{array}$ & Moy. & E.T. & $\begin{array}{c}\text { Nb. } \\
\text { d'agnelles }\end{array}$ & Moy. & E.T. \\
\hline $\mathrm{CP} 2$ & 60 & $2,86^{a b}$ & 0,77 & 50 & $3,12^{a}$ & 0,41 & 10 & $2,55^{b}$ & 0,93 \\
\hline $\mathrm{C} 3$ & 60 & $2,69^{a}$ & 0,84 & 38 & $3,23^{b}$ & 0,49 & 22 & $2,40^{a}$ & 0,95 \\
\hline P4 & 60 & $2,83^{a}$ & 0,82 & 41 & $2,80^{a}$ & 0,47 & 19 & $2,24^{b}$ & 0,84 \\
\hline T5 & 60 & $2,82^{a}$ & 0,83 & 29 & $2,30^{b}$ & 0,39 & 31 & $2,06^{b}$ & 0,86 \\
\hline Moyenne & 240 & $2,80^{a}$ & 0,81 & 158 & $2,92^{a}$ & 0,55 & 82 & $2,26^{b}$ & 0,89 \\
\hline \multicolumn{10}{|c|}{${ }^{1}$ Ecart-type } \\
\hline \multicolumn{10}{|c|}{${ }^{2}$ Complémentation alimentaire et traitements prophylactiques } \\
\hline \multicolumn{10}{|c|}{${ }^{3}$ Complémentation alimentaire sans traitements prophylactiques } \\
\hline \multicolumn{10}{|c|}{${ }^{4}$ Traitements prophylactiques uniquement } \\
\hline${ }^{5}$ Témoin & & & & & & & & & \\
\hline
\end{tabular}

\section{Tableau V}

Comparaisons de quelques paramètres métaboliques des agnelles avant leur mort au cours de l'essai et des agnelles vivantes

\begin{tabular}{|c|c|c|c|c|c|c|c|c|}
\hline Lot & & $P^{1}$ & C & 2 & $\mathbf{P}$ & & & † \\
\hline $\begin{array}{l}\text { Statut des agnelles par lot } \\
\mathrm{Nb} \text {. de prélèvements }\end{array}$ & $\begin{array}{c}\text { Mortes } \\
3\end{array}$ & $\begin{array}{l}\text { Vivantes } \\
154\end{array}$ & $\begin{array}{c}\text { Mortes } \\
5\end{array}$ & $\begin{array}{l}\text { Vivantes } \\
139\end{array}$ & $\begin{array}{c}\text { Mortes } \\
4\end{array}$ & $\begin{array}{l}\text { Vivantes } \\
148\end{array}$ & $\begin{array}{c}\text { Mortes } \\
13\end{array}$ & $\begin{array}{c}\text { Vivantes } \\
124\end{array}$ \\
\hline $\begin{array}{l}\text { U rée g/l } \\
\text { Glucose } g / l \\
\text { Hémoglobine \% } \\
\text { Hématocrite \% }\end{array}$ & $\begin{array}{c}0,22 \\
0,61^{a} \\
5,63^{a} \\
15,7^{a}\end{array}$ & $\begin{array}{c}0,35 \\
0,63^{a} \\
9,48^{b} \\
27,6^{b}\end{array}$ & $\begin{array}{c}0,29 \\
0,58^{a} \\
5,74^{a} \\
13,1^{a}\end{array}$ & $\begin{array}{c}0,32 \\
0,63^{a} \\
9,35^{b} \\
27,8^{b}\end{array}$ & $\begin{array}{c}0,10 \\
0,47^{b} \\
6,55^{a} \\
16,8^{a}\end{array}$ & $\begin{array}{c}0,20 \\
0,60^{b} \\
9,32^{b} \\
27,2^{b}\end{array}$ & $\begin{array}{c}0,25 \\
0,45^{b} \\
6,68^{a} \\
15,6^{a}\end{array}$ & $\begin{array}{c}0,25 \\
0,60^{\mathrm{a}} \\
8,60^{\mathrm{b}} \\
25,8^{\mathrm{b}}\end{array}$ \\
\hline $\begin{array}{l}{ }^{1} \text { Complémentation alimentaire et tra } \\
{ }^{2} \text { Complémentation alimentaire sans } \\
{ }^{3} \text { Traitements prophylactiques unique } \\
{ }^{4} \text { Témoin }\end{array}$ & $\begin{array}{l}\text { nts prophyla } \\
\text { ments proph }\end{array}$ & $\begin{array}{l}\text { ctiques } \\
\text { ylactiques }\end{array}$ & & & & & & \\
\hline
\end{tabular}

\section{DISCUSSION}

Cette étude a montré que des symptômes, particulièrement le jetage, sont souvent observés en saison sèche froide, et les diarrhées en saison des pluies. Les mortalités sont cependant plus nombreuses en saison des pluies. L'évolution de la NEC permet d'apprécier l'état nutritionnel des agnelles. Leur état devient critique lorsque la NEC est inférieure à 2,5 sur une échelle de notation de 1 à 4. Les ovins, au sortir d'une période de sous-alimentation en saison sèche, sont affaiblis et deviennent plus vulnérables aux infestations parasitaires du début de saison des pluies. Les observations d'Awa et Njoya (non publiées) au cours de la même expérience faites à partir de 30 autopsies, ont montré que les parasites digestifs tels que Haemonchus contortus et Moniezia expanza étaient responsables de 75 p. 100 des mortalités des agnelles. Les résultats de cette enquête sont en accord avec ceux de Tillard et coll. (12) en zone soudanienne du Sénégal qui indiquaient que les mortalités liées au parasitisme digestif chez les ovins étaient cinq fois plus importantes que celles liées à la pathologie infectieuse. Dans cette étude, les nombreux cas de boiterie $(65,6$ p. 100) enregistrés dans les lots $\mathrm{C}$ et $\mathrm{T}$ (lots sans traitements ni prophylaxie) étaient surtout dus aux tiques. Le grand nombre de cas observés dans le lot $\mathrm{T}$ (39,3 p. 100) aurait été aggravé par la sous-alimentation. Une épidémie de gale s'était abattue dans le lot $\mathrm{C}$ sans toutefois être fatale pour les agnelles. De nombreux cas d'ecthyma ont été observés dans le lot $\mathrm{CP}$, sans que l'on puisse expliquer son origine.

Les traitements prophylactiques, sans toutefois influencer de façon significative l'apparition des maladies, ont permis une réduction importante du quotient de mortalité. Soixante-et-un pour cent des mortalités étaient enregistrées dans les lots ne recevant pas de traitements sanitaires (lots C et T). Au Nigeria, Opasina (7) a enregistré une baisse de la mortalité du même ordre sur des chèvres, grâce à un détiquage mensuel et une vaccination annuelle contre la PPR. Omeke (6) indiquait dans la même région du Nord du Nigeria qu'on peut préconiser deux vermifugations des ovins, l'une en début des pluies et l'autre en fin des pluies, afin de réduire la mortalité. 
Dans cette étude, les traitements prophylactiques ont été beaucoup plus efficaces dans la réduction du quotient de mortalité lorsqu'ils ont été couplés à une complémentation protéique (16,7 p. 100 dans le lot $\mathrm{CP}$ contre 31,7 p. 100 dans le lot $\mathrm{P}$ ), soit une réduction de la mortalité de l'ordre de 50 p. 100. Les ovins morts dans le lot T présentaient des signes évidents de parasitisme gastro-intestinal et de sous-alimentation (amaigrissement et poils piqués). La baisse de l'urémie et de la glycémie chez les agnelles évoluant vers la mort témoigne de leur état carentiel généralisé, tant sur le plan azoté qu'énergétique. Cela concorde avec les observations de Thys et Vercruysse (10) qui ont observé que le parasitisme dépendait également de l'état nutritionnel de l'animal. Au Nord-Cameroun, le problème alimentaire se pose avec beaucoup d'acuité, surtout en fin de saison sèche lorsque la teneur protéique et énergétique des herbacées est extrêmement faible, et que celles-ci ne peuvent même pas couvrir les besoins d'entretien des animaux. Cette alimentation pauvre les fragilise. Ils deviennent anémiques et succombent facilement lorsqu'ils sont infestés de parasites gastrointestinaux lors du retour des pluies.

Malgré les nombreux cas de jetage (145 cas) au cours de la saison sèche (novembre à mars), la mortalité a été plus faible à cette période, ce qui laisse croire que l'infestation par les parasites digestifs, aggravée par la malnutrition, semble être le principal facteur de mortalité des ovins dans cette étude. Ces résultats concordent avec ceux de Tillard (11) indiquant la fréquence très élevée de jetage, sans toutefois être fatale, chez les ovins en zone sahélienne du Sénégal. Une prise en compte de la morbidité clinique seule peut entraîner une nette sous-estimation de l'importance d'autres facteurs dans l'occurrence de mortalité. Cela est conforme à l'idée selon laquelle c'est la conjonction de plusieurs facteurs qui entraîne une pathologie fatale $(3,4)$. Au Nord-Cameroun, s'occuper spécifiquement de l'alimentation ou de la vaccination contre la PPR sans tenir compte du parasitisme gastro-intestinal ne peut qu'apporter une réduction relativement faible de la mortalité et présente un intérêt économique incertain. Par ailleurs, une campagne de lutte contre les helminthoses $(6,9,10)$ ne peut être économiquement avantageuse que lorsque le statut nutritionnel des animaux est considéré.

Le jetage est le principal symptôme clinique observé chez les ovins, mais les infestations par des parasites gastro-intestinaux et la sous-alimentation ont été les facteurs favorisant les fortes mortalités enregistrées dans les troupeaux. La complémentation protéique et la prophylaxie sanitaire (détiquage, vermifugation et vaccination contre la PPR) réduit la mortalité, ce qui est classique, cependant de meilleurs résultats sont obtenus par la synergie des deux actions. Les besoins alimentaires des ovins ne pouvant être couverts par les pâturages naturels, la complémentation a été un facteur améliorant l'effet des traitements sanitaires sur la viabilité des agnelles dans cette étude, indiquant ainsi qu'une approche multiforme est nécessaire dans le contrôle de la mortalité. La pratique d'une complémentation protéique associée à une vermifugation et une vaccination annuelle contre la PPR dès le retour des pluies, et un déparasitage externe dès qu'il y a des tiques sur les animaux devrait être encouragée en vue d'une maîtrise des fortes mortalités observées dans les élevages ovins en zone soudano-sahélienne du Nord-Cameroun. Son application en milieu paysan devrait également permettre de mieux appréhender l'influence d'autres facteurs tels que l'habitat, l'éleveur et la zone d'élevage, dans l'apparition des symptômes cliniques et la mortalité des ovins. La prise en compte ultérieure des performances de reproduction permettra de préciser l'intérêt économique de ce programme de complémentation et de prophylaxie.

\section{Remerciements}

Cette étude a été financée par le Projet régional de recherches sur les petits ruminants (Cameroun - Niger - Tchad). Nous remercions MM. Njifotié, Kammengni, Nassourou et Awahmukalah de la Station polyvalente de Garoua, pour la collecte des données, Dr Daniel Bourzat (CIRAD-EMVT, Nouvelle Calédonie), pour l'appui logistique, et le Dr Bernard Faye (CIRAD-EMVT, Montpellier, France) pour ses conseils et la relecture de cet article.

\section{BIBLIO GRAPHIE}

1. CARDINALE E., NGO TAMA A.C., NJOYA A., 1996. L'élevage des petits ruminants au Nord-Cameroun. In : Agricultures des savanes du Nord-Cameroun, Atélier d'échange, 25-29 novembre 1996, Garoua, Cameroun.

2. FAU GERE O., FAU GERE B., MERLIN P., DOCKES C., PERROT C., 1988. L'élevage traditionnel des petits ruminants dans la zone de Kolda (Haute Casamance) : Référentiel technico-économique (données recueillies dans vingt villages de 1984 à 1987). Dakar, Sénégal, ISRA/LNERV, 187 p. (D ocument de travail no 1/Kolda)

3. FAYE B., LEFEVRE P.C., LANCELOT R., QUIRIN R., 1994. Ecopathologie animale. M éthodologie et applications en milieu tropical. Maisons-Alfort, France, CIRAD-EMVT, 119 p.

4. GANIERE J.P., ANDRE-FO NTAINE G., DROU IN P., FAYE B., MADEC F., ROSNER G., FOURICHON G., WANG B., TILLON J.P., 1991. L'écopathologie : une méthode d'approche de la santé animale. INRA Prod. Anim., 4 : 247-256.

5. NJOYA A. Protein supplementation of grazing cattle in the semi-arid zone of Cameroon. Trop. Anim. Health Prod. (sous presse)

6. OMEKE B.C.O., 1988. Evaluation of three strategic prophylactic programmes against helminthiasis of traditionally managed West African D warf sheep and goat in Nigeria. Br. vet J., 6: 590-595.

7. OPASINA B.A., 1985. Disease constraints on productivity of village goats in Southwest Nigeria. Ibadan, Nigeria, ILCA. (Humid zone programme document $\mathrm{N} 0.5$ )

8. SAS/STAT: Statistical Analytical Systems, 1990. Vers. 6, U ser's guide: Statistics. Cary, NC, USA, SAS Institute Inc.

9. SCHILLHORN VAN VEEN T.W., OGUNSUSI R.A., 1978. Epidémiologie et contrôle de I'helminthiase gastro-intestinale chez les moutons au Nigeria. Session générale du comité I'O IE, Paris, 22-27 mai 1978. Paris, France, OIE. (Rapport n 106 bis)

10. THYS E., VERCRUYSSE J., 1990. Est-il encore opportun de préconiser la vermifugation systématique des petits ruminants d'Afrique sahélo-soudanienne contre les nématodes gastro-intestinaux ? Revue Elev. M éd. vét. Pays trop., 43 : 187-191.

11. TILLARD E., 1994. Caractérisation de l'atteinte par les affections respiratoires des troupeaux d'ovins de la zone sahélienne au Sénégal. Vet. Res., 25 : 348-354.

12. TILLARD E., FAU GERE O., FAU GERE B., 1992. Evaluation technicoéconomique de prophylaxies chez les petits ruminants au Sénégal : régionalisation des interventions de protection sanitaire. In : Actes $7 \mathrm{e}$ Conférence international des institutions de médecine vétérinaire tropicale, Yamoussoukro, Côte d'Ivoire. Berlin, Allemagne, DSE, Maisons-Alfort, France, CIRAD-EMVT, p. 519-528.

Reçu le 24.4.95, accepté le 16.12.97 


\section{Summary}

N joya A., Awa N.D., Bouchel D. Influence of feed supplementation and disease prophylaxis on the viability of Fulbe sheep in North-Cameroon

Four groups of 60 weaner ewes aged 3 to 5 months (CP group: feed supplementation, vaccination against peste des petits ruminants, anthelmintic and external antiparasitic treatments; $C$ group: feed supplementation without prophylaxis nor treatment; $P$ group: prophylaxis and treatment, but no feed supplementation; T group: control) were formed in April 1993 in order to study the effects of feed supplementation and prophylactic treatment on their viability. Feed supplementation was made of $200 \mathrm{~g} / \mathrm{head} /$ day cotton seed cakes and mineral licking blocks. The prophylactic treatment consisted in two internal antiparasitic treatments at the beginning and the end of the rainy season and an external treatment whenever ticks were seen on the ewes. Clinical observations and body state notations were recorded over a one year period. $\mathrm{N}$ asal discharge, lameness and diarrhea were the main clinical signs observed $(50.9,15.2$ and $13.0 \%$, respectively). The annual mortality rates were $16.7,37.3,31.7$ and $52.5 \%$ for groups $C P, C, P$ and $T$, respectively. Supplementation and prophylaxis significantly reduced mortality $(p<0.05)$. A sharp rise in mortality rates was noticed with the coming of the rains. Gastrointestinal nematode infestation and undernutrition were the leading factors of mortality. The strategy of protein supplementation and prophylaxis might be encouraged to reduce the high mortality, the main restricting factor in sheep livestock production in N orth Cameroon.

Key words: Fulbe sheep - Ewe - Supplementary feeding Disease control - Viability - W et season - Cameroon.

\section{Resumen}

Njoya A., Awa N.D., Bouchel D. Influencia del complemento y la profilaxis sobre la viabilidad de los ovinos Foulbé, al Norte de Camerún

Cuatro lotes de 60 ovejas de 3 a 5 meses (lote CP: complemento, vacunación contra la peste de los pequeños rumiantes y desparisitación; lote $\mathrm{C}$ : complemento sin profilaxis; lote $\mathrm{P}$ : profilaxis sin complemento; lote $\mathrm{T}$ : control) se constituyeron en abril de 1993, con el fin de estudiar el efecto del complemento alimenticio y de tratamientos sanitarios sobre la viabilidad. El complemento consistió en $200 \mathrm{~g}$ de torta de algodón por cabeza y por día y un complemento mineral en bloc para lamer. Los tratamientos sanitarios consistieron en dos desparasitaciones internas al inicio y al fin de las lluvias y una desparasitación externa coda vez que se observó la presencia de garrapatas. Los animales fueron objeto de un seguimiento clínico y de un registro del progreso del estadio corporal en el año. Ilujas, diarreas y cojeras representaron los principales signos clínicos observados (50,13,0 y 15,2\% respectivamente). Le cociente anual de mortalidad fue de 16,7, 37,3, 31,7 y $52,5 \%$ respectivamente para los lotes $C P, C, P$ y $T$. El complemento y la profilaxis permitieron una reducción notable de la mortalidad ( $p<0,05)$. La mortalidad aumentó considerablemente con el regreso de las Iluvias. Las infestaciones por parásitos gastrointestinales y la sub-alimentación constituyeron los principales factores favorecedores de la mortalidad. Esta estrategia de complemento proteico y de profilaxis puede recomendarse para reducir las altas mortalidades, principal factor limitante de la productividad de los criaderos bovinos en N orte-Camerún.

Palabras clave: Ovino Foulbe - Oveja - Alimentación complementaria - Control de enfermedad - Viabilidad Estación húmeda - Camerún. 